\title{
Chemovirotherapy for pancreatic cancer: Gemcitabine plus oncolytic measles vaccine virus
}

\author{
VERENA MAY ${ }^{1}$, SUSANNE BERCHTOLD ${ }^{2,3}$, ALEXANDER BERGER $^{4}$, SASCHA VENTURELLI $^{5}$, \\ MARKUS BURKARD ${ }^{5}$, CHRISTIAN LEISCHNER ${ }^{5}$, NISAR P. MALEK ${ }^{1}$ and ULRICH M. LAUER ${ }^{2,3}$
}

\begin{abstract}
Departments of ${ }^{1}$ Internal Medicine I (Gastroenterology, Gastroenterologic Oncology, Hepatology, Infectiology and Geriatric Medicine) and ${ }^{2}$ Internal Medicine VIII (Medical Oncology and Pneumology), University Hospital Tuebingen, D-72076 Tuebingen; ${ }^{3}$ German Cancer Consortium (DKTK), DKFZ Partner Site Tuebingen, Interfaculty Institute of Biology, D-72076 Tuebingen; ${ }^{4}$ Boehringer Ingelheim Pharma GmbH and Co. KG, D-88397 Biberach/Riss;

${ }^{5}$ Department of Vegetative and Clinical Physiology, University Hospital Tuebingen, D-72076 Tuebingen, Germany
\end{abstract}

Received October 16, 2018; Accepted March 19, 2019

DOI: $10.3892 / 01.2019 .10901$

\begin{abstract}
Oncolytic virotherapy with vaccine viruses employs replicative vectors, which quite selectively infect tumor cells leading to massive virus replication followed by subsequent profound tumor cell death (oncolysis). Measles vaccine virus $(\mathrm{MeV})$ has already shown great oncolytic activity against different types of cancers, including pancreatic cancer. Gemcitabine is a first line chemotherapeutic drug used for pancreatic cancer in palliative treatment plans. Furthermore, this drug can be used to induce senescence, a permanent cell cycle arrest, in tumor cells. In our preclinical work, three well-characterized immortalized human pancreatic cancer cell lines were used to investigate the combinatorial effect of MeV-based virotherapy together with the chemotherapeutic compound gemcitabine. Viability assays revealed that the combination of only small amounts of $\mathrm{MeV}$ together with subtherapeutic concentrations of gemcitabine resulted in a tumor cell mass reduction of $>50 \%$. To further investigate the replication of the oncolytic $\mathrm{MeV}$ vectors under these distinct combinatorial conditions, viral growth curves were generated. As a result, viral replication was found to be only slightly diminished in the presence of gemcitabine. As gemcitabine induces senescence, the effect of $\mathrm{MeV}$ on that phenomenon was explored using a senescence-associated $\beta$-galactosidase assay. Notably, gemcitabine-induced tumor cell senescence
\end{abstract}

Correspondence to: Professor Ulrich M. Lauer, Department of Internal Medicine VIII (Medical Oncology and Pneumology), University Hospital Tuebingen, Otfried-Mueller-Street 10, D-72076 Tuebingen, Germany

E-mail: ulrich.lauer@uni-tuebingen.de

Abbreviations: hpi, hours post infection; $\mathrm{MeV}$, measles vaccine virus; MOI, multiplicity of infection (ratio infectious particles/cell); $\mathrm{OV}$, oncolytic virus; $\mathrm{PFU}$, plaque forming unit

Key words: oncolytic virotherapy, pancreatic cancer, oncolytic measles vaccine virus, chemovirotherapy, gemcitabine, senescence was not impaired by MeV. Accordingly, the chemovirotherapeutic combination of gemcitabine plus oncolytic $\mathrm{MeV}$ constitutes a novel therapeutic option for advanced pancreatic carcinoma that is characterized by the mutual improvement of the effectiveness of each therapeutic component.

\section{Introduction}

First line therapy for locally advanced and metastasized pancreatic cancer comprises the chemotherapeutic compound gemcitabine, which belongs to the class of nucleoside analogues $(1,2)$. However, resistance towards gemcitabine is common and gives one explanation for the poor prognosis being associated with its monotherapeutic usage. Add-ons of other drugs such as the tyrosinekinase-inhibitor (TKI) erlotinib (EGFR-TKI) or nab-paclitaxel improve survival only slightly and are affiliated with additional side effects (3).

Oncolytic viruses (OVs) constitute replication-competent particles, which are mostly non-cytotoxic for normal tissues exhibiting intact anti-viral immune defense signaling. In contrast, these OVs are able to selectively infect, replicate within and lyse tumor cells mostly displaying impaired anti-viral interferon pathways (4). Newly released viral particles then can infect hitherto uninfected neighboring tumor cells which lead to subsequent waves of oncolytic tumor cell death and induction of a profound systemic anti-tumoral immune response (5-7).

Numerous OVs are currently under investigation for pancreatic cancer therapy and many clinical trials are being performed (8-11). We here employed measles vaccine virus (MeV)-a negative-stranded RNA virus, belonging to the paramyxovirus group. $\mathrm{MeV}$ has an excellent safety record as it has been used as a vaccine for more than 50 years. With regard to its oncolytic efficiency, a first case of durable complete remission after a single systemic $\mathrm{MeV}$ treatment was reported recently for a patient suffering from therapy-refractory multiple myeloma (12). In preclinical models, pancreatic cancer was shown to be susceptible to $\mathrm{MeV}$ as well (13-16). Importantly, $\mathrm{MeV}$ recently also was found to infect and lyse gemcitabine-resistant pancreatic carcinoma cells (14). 
In the present study, we further investigated the influence of gemcitabine on the oncolytic efficiency of $\mathrm{MeV}$ in more human pancreatic cancer cell lines. We also characterized the influence of gemcitabine on replication of $\mathrm{MeV}$, as viral replication is postulated to be a prerequisite for effectiveness of oncolysis and subsequent rounds of infection. In a previous study we could show that senescent tumor cells, such as the human hepatoma cell line HepG2, the human mammary gland cancer cell line MCF7 and the pancreatic cancer cell line MIA $\mathrm{PaCa}-2$, can be infected and lysed by $\mathrm{MeV}$ more efficiently than their non-senescent counterparts (17). Accordingly, viral replication was even more efficient in senescent cells. Finally, we wanted to determine whether gemcitabine-induced tumor cell senescence could be impaired by previous infection with $\mathrm{MeV}$ or not.

\section{Materials and methods}

Cell culture/viral stocks. MIA PaCa-2, PANC-1, and BxPC-3 (all human pancreatic carcinoma) and Vero (African green monkey kidney) cells were obtained from the German Collection of Microorganisms and Cell Cultures (DSMZ, Braunschweig, Germany) and cultured in Dulbecco's modified Eagle's medium (Biochrom, Berlin, Germany) with 10\% fetal calf serum (PAA, Pasching, Austria) in a humidified incubator $\left(37^{\circ} \mathrm{C}, 5 \% \mathrm{CO}_{2}\right)$. Our prototypic suicide gene-armed vector $\mathrm{MeV}-\mathrm{SCD}$ was generated from a commercially available original monovalent vaccine batch of $\mathrm{MeV}$ strain Mérieux (Sanofi-Pasteur, Leimen, Germany), as described recently (18). Recombinant $\mathrm{MeV}$ carrying a green fluorescent marker protein (MeV-GFP) was generated by real time-polymerase chain reaction cloning from an original vaccine batch of the $\mathrm{MeV}$ Schwarz strain (Mérieux, Sanofi-Pasteur, Lyon, France).

Substance. Gemcitabine was obtained from LC Laboratories (Woburn, MA, USA) and solved in dimethyl sulfoxide.

Cell viability assays. Cells were plated in 24-well plates $\left(4 \times 10^{4}\right.$ cells per well) and allowed to adhere for $24 \mathrm{~h}$. Infection with $\mathrm{MeV}$ was performed after washing cells once with phosphate buffered saline (PBS) in Opti-MEM serum-reduced medium (Gibco; Thermo Fisher Scientific, Inc., Waltham, MA, USA) for $3 \mathrm{~h}$ at $37^{\circ} \mathrm{C}$. Virus was allowed to infect tumor cells for $3 \mathrm{~h}$. Then the medium was changed with the addition of the chemotherapeutic compound (i.e., gemcitabine) (Fig. 1A). Cell viabilities were determined after an incubation time of $72 \mathrm{~h}$, employing the Suforhodamine B assay (SRB, measuring remaining cell mass) (19) as well as the MTT assay (measuring cellular enzyme activity, a yellow-colored tetrazole (3-(4,5-dimethylthiazol-2-yl)-2,5-diphenyltetrazolium bromide, which is reduced to formazan (purple color) by living cells) (20). For SRB assay, cells were fixed with cold $10 \%$ trichloroacetic acid (TCA) and dried at $40^{\circ} \mathrm{C}$. Cells were stained with SRB staining solution $(0.4 \%$ Sulforhodamine B in $1 \%$ acetic acid), and after drying, stain was solubilized in $10 \mathrm{mM}$ Tris base, $\mathrm{pH}$ 10.5. Optical density was measured at a wavelength of $550 \mathrm{~nm}$ in a microtiter plate reader. For MTT assay, cells were washed with warm PBS and stained with MTT staining solution (MTT $2.5 \mathrm{mg} / \mathrm{ml}$ solubilized in
DMEM without phenol red). Then plates were incubated at $37^{\circ} \mathrm{C}$ for $2 \mathrm{~h}$. After incubation time, the staining solution was removed and plates were frozen at $-20^{\circ} \mathrm{C}$. For measurement, stained cells were solubilized in $\mathrm{MTT}$ solvent $(3.7 \% \mathrm{HCl}$ in isopropanol). Optical density was measured at a wavelength of $570 \mathrm{~nm}$ (reference wave length $650 \mathrm{~nm}$ ).

Virus growth curves. To measure viral replication comparing MeV-SCD alone with the combination of MeV-SCD and gemcitabine, virus growth curves were generated. Infection was performed in analogy to the cell viability assays with the same multiplicities of infection (MOIs; ratios of infectious viral particles/tumor cells) of MeV-SCD and concentrations of gemcitabine using six-well plates. Three hours post infection (hpi) the inoculum was removed and cells were washed three times with PBS. Then, $1 \mathrm{ml}$ of medium with or without gemcitabine was added. Supernatants and cells were harvested at indicated time points (Fig. 2). Viral titers were determined according to the method of Kärber and Spearman $(21,22)$. Samples of either cell suspensions undergoing extractions of the cellular contents (graphs to the left in Fig. 3: Represented by 'cell associated virus') or cell culture medium supernatants (graphs to the right in Fig. 3: 'Released virus') were used to re-infect Vero cells with dilution series. After 96 h, Vero cells were fixed with $4 \%$ formaldehyde and stained with fluorescent antibodies (primary antibody: Anti-MeV-NP, clone 120; no. 95040312; ECACC, Salisbury, UK), secondary antibody: Goat anti-mouse (Alexa Fluor 546; Invitrogen; Thermo Fisher Scientific, Inc.), both diluted 1:1,000 in TBS-Tween). Cells were analyzed via fluorescence microscopy.

Senescence assay. For senescence assay, cells were seeded in 6 -well plates $\left(2 \times 10^{4}\right.$ cells per well). For fixation and staining, the Senescence Cells Histochemical Staining kit (Sigma-Aldrich; Merck KGaA, Darmstadt, Germany) was used. Fixed cells were treated with staining solution containing $2.5 \%$ X-Gal (5-bromo-4-chloro-3-indolyl- $\beta$-D-galactopyrano side) and incubated for 12 to $16 \mathrm{~h}$ at $37^{\circ} \mathrm{C}$ in a carbon dioxide depleted atmosphere. Senescence-associated- $\beta$-galactosidase (SA- $\beta-$ Gal) positive cells appearing light blue in bright field microscopy were calculated as percentage of total cell counts using an Olympus IX50 inverted microscope (Olympus, Center Valley, PA, USA).

Detection of GFP expression. To illustrate viral infection microscopically, infection was performed using a $\mathrm{MeV}$ encoding a green fluorescent marker protein (MeV-GFP). To detect fluorescence, an Olympus IX50 microscope was used.

Statistical analyses. Results in the figures are expressed as mean values with their standard errors. One-way analysis of variance with Bonferroni's multiple comparison test was used to determine the statistical differences when comparing multiple groups. An unpaired t-test, confidence interval $95 \%$ and two-tailed, was used to determine statistical differences between two groups compared once. All analyses were performed using GraphPad Prism v.4.03 (GraphPad Software, Inc., La Jolla, CA, USA). According to this analyses, a P>0.05 was marked as not significant (n.s.) and a $\mathrm{P}<0.05$ was considered to indicate a statistically significant difference. 

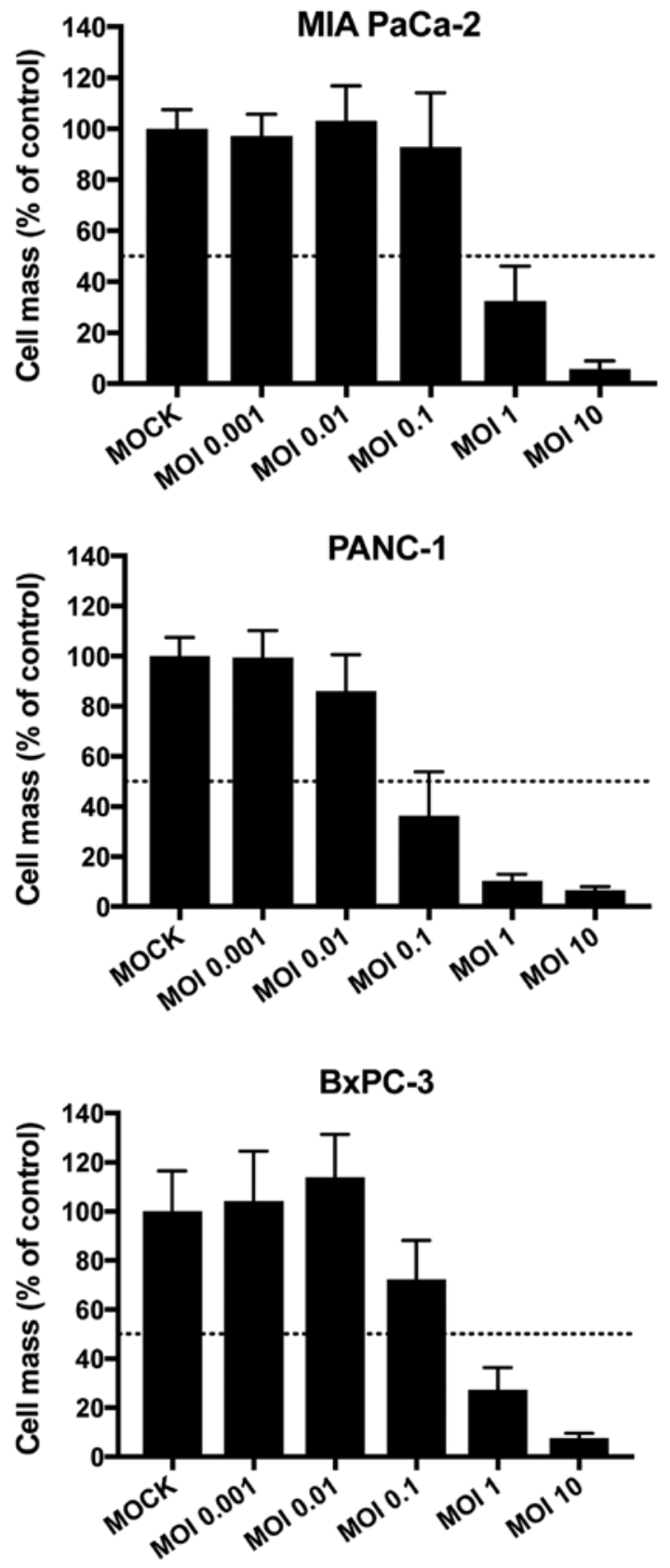

Figure 1. Susceptibility of pancreatic cancer cell lines to MeV-mediated oncolysis. Cells were infected with oncolytic measles vaccine virus $\mathrm{MeV}-\mathrm{SCD}$ at the indicated MOIs. At $72 \mathrm{~h}$ post infection the remaining cell masses were determined by Sulforhodamin B viability assays; $50 \%$ threshold is marked by a dotted line. Data are presented as the mean \pm standard deviation of three independent experiments. MeV, measles vaccine virus, MOIs, multiplicities of infection.

\section{Results}

Efficiency of chemovirotherapy. To investigate a combinatorial effect of $\mathrm{MeV}$ and gemcitabine, drug concentrations of both agents were chosen low enough to aim for a remaining cell viability of more than $50 \%$ in comparison to a non-treated MOCK-control when applied as single agents (Fig. 2B). Two different cell viability assays were performed: i) Sulforhodamin B (SRB) assays, measuring remaining cell masses, and ii) MTT assays, measuring cellular enzyme activity as a second option.
To first determine the adequate dosages for both chemotherapy and virotherapy, tumor cells were infected with increasing multiplicities of infection (MOIs) of MeV-SCD ranging from 0.001 to 10 or treated with different concentrations of gemcitabine in monotherapy. The objective was to find dosages leading to reductions of tumor cell masses between 15 and $45 \%$ in monotherapy. The preliminary finding experiments of $\mathrm{MeV}$ are shown in Fig. 1. The ultimate dosages of both $\mathrm{MeV}$ and gemcitabine are depicted within Fig. 2. Beneath the preliminary experiments depicted in Fig. 1, further experiments were performed to determine the optimal MOIs for the combinatorial approach with steps in between the initially chosen MOIs (Fig. 2B). When comparing these two sets of experiments (Fig. 1 and Fig. 2B) slight differences in tumor cell viabilities at $72 \mathrm{~h}$ post infection were detected which could be explained by biological variations. As a result of both SRB and MTT assays, cell viabilities were found to undercut the $50 \%$ threshold in all tumor cell lines when MeV-SCD and gemcitabine were combined (Fig. 2B), which constitutes a considerable cytotoxic effect in contrast to the rather low efficiencies of the therapeutics alone ('monotherapeutic' results).

In MIA PaCa-2 (Fig. 2B, upper panel), cell viabilities of the MeV-SCD plus gemcitabine combination therapy even were found to be reduced to less than $30 \%$ of the controls, whereas cell viabilities did not undercut $70 \%$ for $\mathrm{MeV}-\mathrm{SCD}$ and $55 \%$ for gemcitabine [when always applying the same amounts of MeV-SCD (MOI 0.4) and gemcitabine (0.03 $\mu \mathrm{M})]$. In PANC-1 (Fig. 2B, middle panel), single agent treatments led to cell viabilities higher than $65 \%(\mathrm{MeV}-\mathrm{SCD}, \mathrm{MOI} 0.075)$ and higher than $75 \%(0.075 \mu \mathrm{M}$ gemcitabine), whereas cell viabilities in dual agent treatments were found to drop down to a range of $40-45 \%$ only. In BxPC-3 (Fig. 2B, lower panel), cell viabilities for MeV-SCD (MOI 0.125) alone were about $80 \%$ and for gemcitabine $(0.02 \mu \mathrm{M})$ alone about $65 \%$; combination of both compounds resulted in cell viabilities of less than $50 \%$.

Taken together, combination therapies significantly reduced cell viabilities were found in all three human pancreatic carcinoma cell lines for the combinatorial approach in comparison to single-agent therapies. Consequently, it became obvious that both agents did not negatively influence the cytotoxic potency of each other in pancreatic cancer cell lines. To confirm this, we investigated replication of MeV-SCD as well as therapy-induced senescence (TIS) caused by gemcitabine in our combinatorial approach.

Chemotherapeutic influence on viral replication. Viral replication within infected tumor cells constitutes one of the most important modes of action of oncolytic virotherapy, as it is indispensable for tumor cell lysis, release of progeny virus particles and further rounds of tumor cell infections. Therefore, we set out to quantify viral replication in absence and presence of gemcitabine. For this purpose, virus growth curves were generated for: i) viral replication within tumor cells (Fig. 3, 'cell associated virus', continuous graphs) and,ii) viral particles being released into supernatants (Fig. 3, 'released virus', dotted graphs). To compare viral replication of $\mathrm{MeV}-\mathrm{SCD}$ alone with the combination of MeV-SCD and gemcitabine, virus growth curves for $\mathrm{MeV}$ alone and for the combination therapy were generated under the same conditions, i.e., MOIs of MeV-SCD 
A Infection

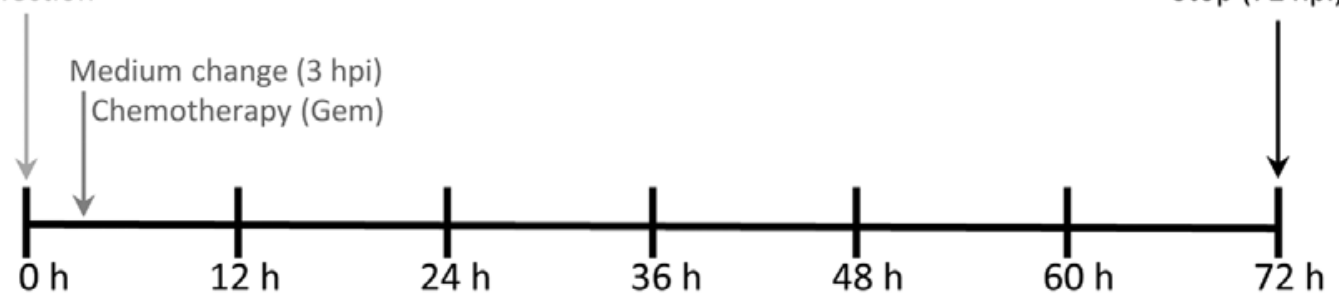

B

MIA PaCa-2
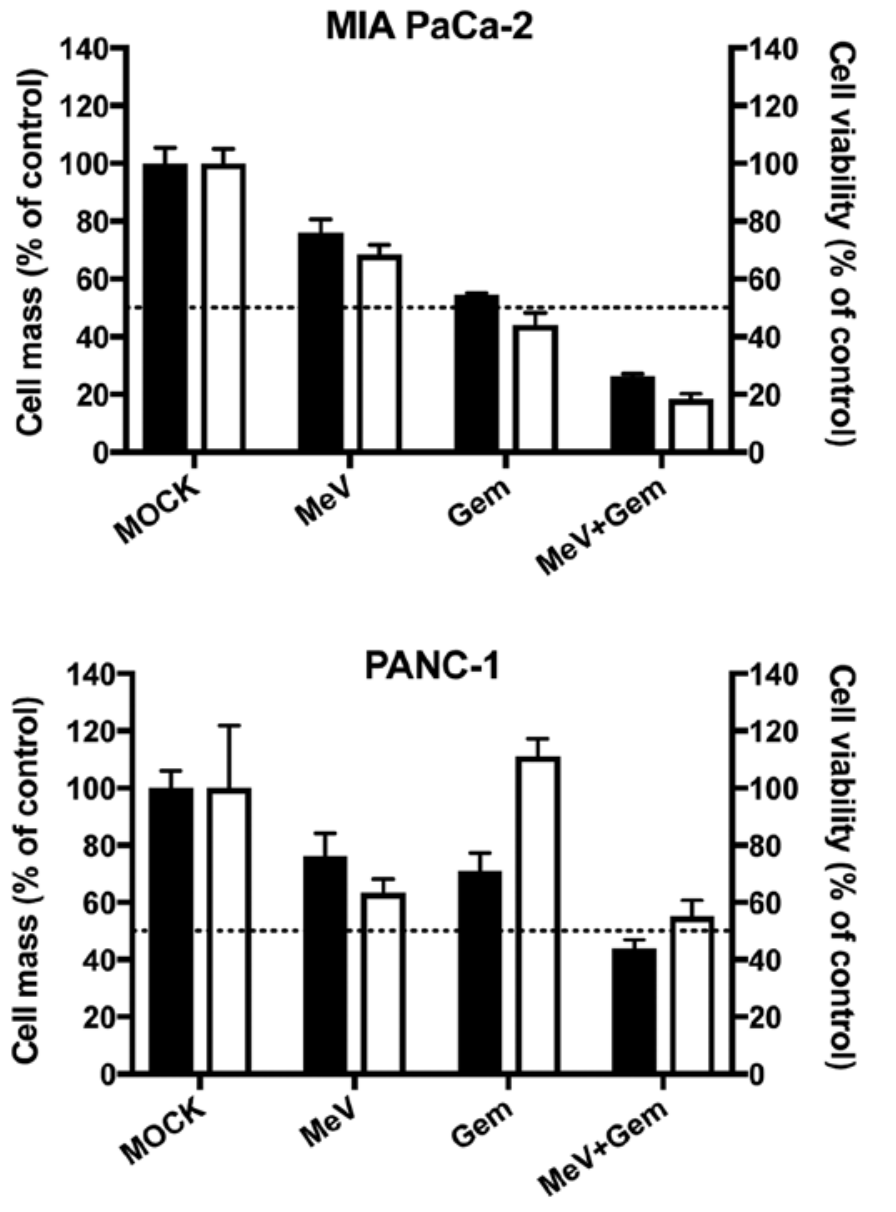

BxPC-3

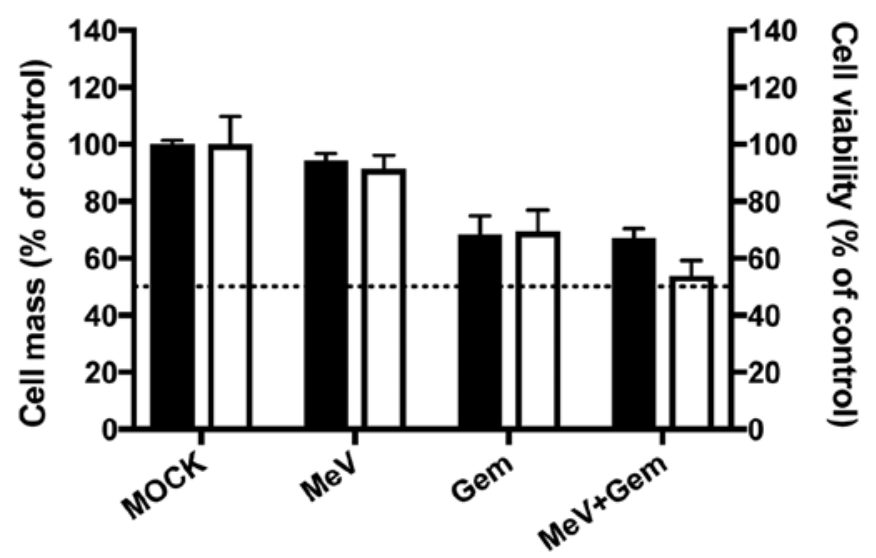

Figure 2. Chemovirotherapy employing Gem together with the oncolytic measles vaccine virus in three pancreatic cancer cell lines. (A) Setting: Tumor cells were infected with MeV-SCD (here denoted as MeV) at $24 \mathrm{~h}$ after plating. Add-on of Gem was performed at 3 hpi when medium was changed. The total incubation time of virus was $72 \mathrm{~h}$. (B) Cell viability was measured using two different assays [SRB (black bars) and MTT (white bars) assays, respectively] and normalized to an uninfected (MOCK-treated) control (set to $100 \%$ cell viability). MOIs of MeV and Gem concentrations were chosen at low enough levels to reduce tumor cell masses $<50 \%$ when used as a single compound. When used in combination, the remaining tumor cell mass was found to be $<5 \%$ in all three tumor cell lines. For MeV, MOIs of 0.4 (MIA PaCa-2), 0.075 (PANC-1) and 0.125 (BxPC-3) were chosen, respectively. For Gem, concentrations of $0.03 \mu \mathrm{M}$ (MIA PaCa-2), $0.075 \mu \mathrm{M}$ (PANC-1) and $0.02 \mu \mathrm{M}$ (BxPC-3) were used, respectively. Data are presented as the mean \pm standard deviation of three independent experiments. GEM, gemcitabine; hpi, hours post infection; MOIs, multiplicities of infection; MeV, measles vaccine virus; SRB, Sulforhodamin B. 


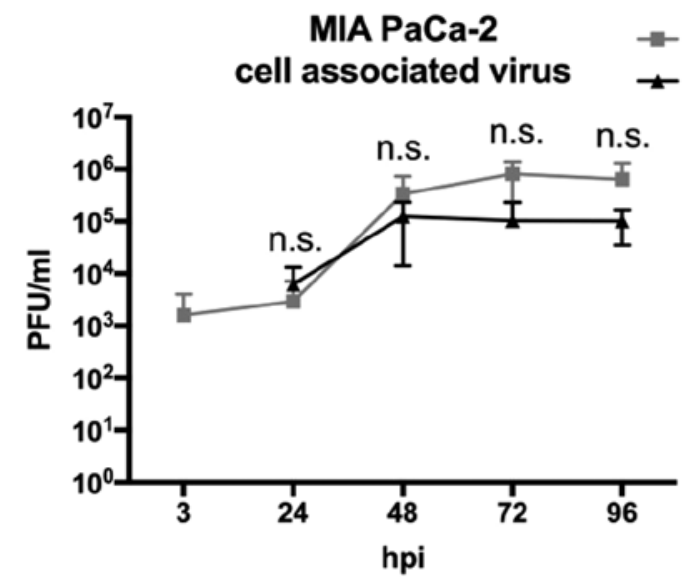

PANC-1

cell associated virus
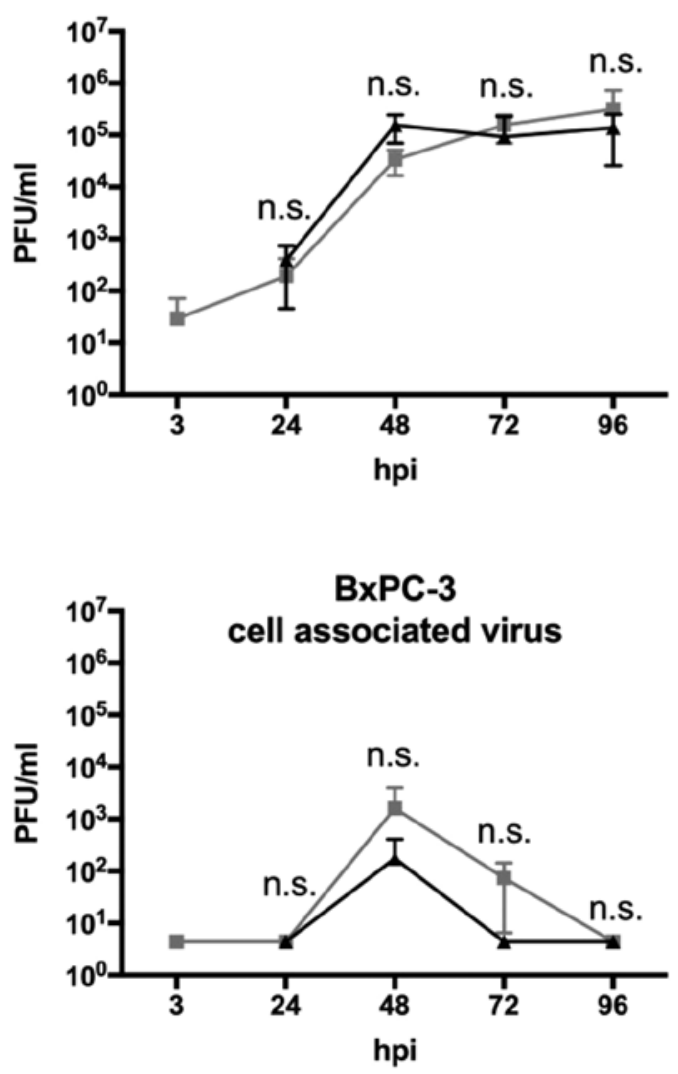

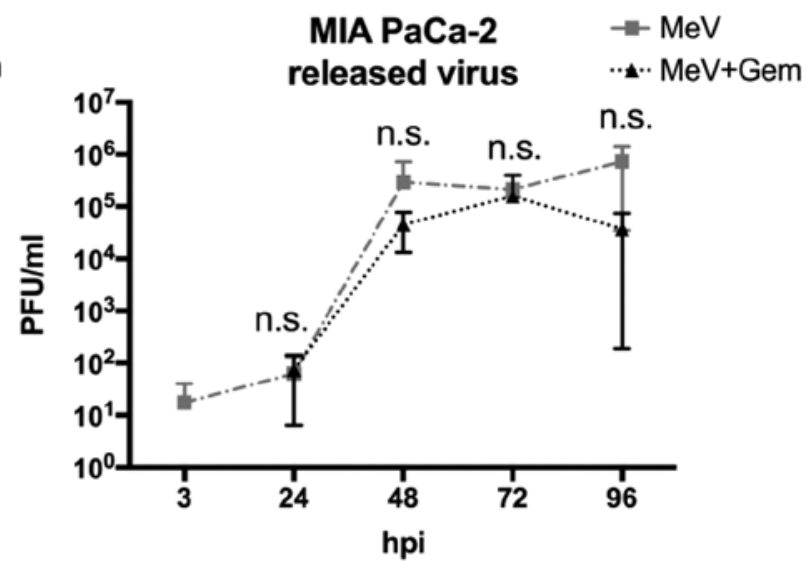

PANC-1 released virus
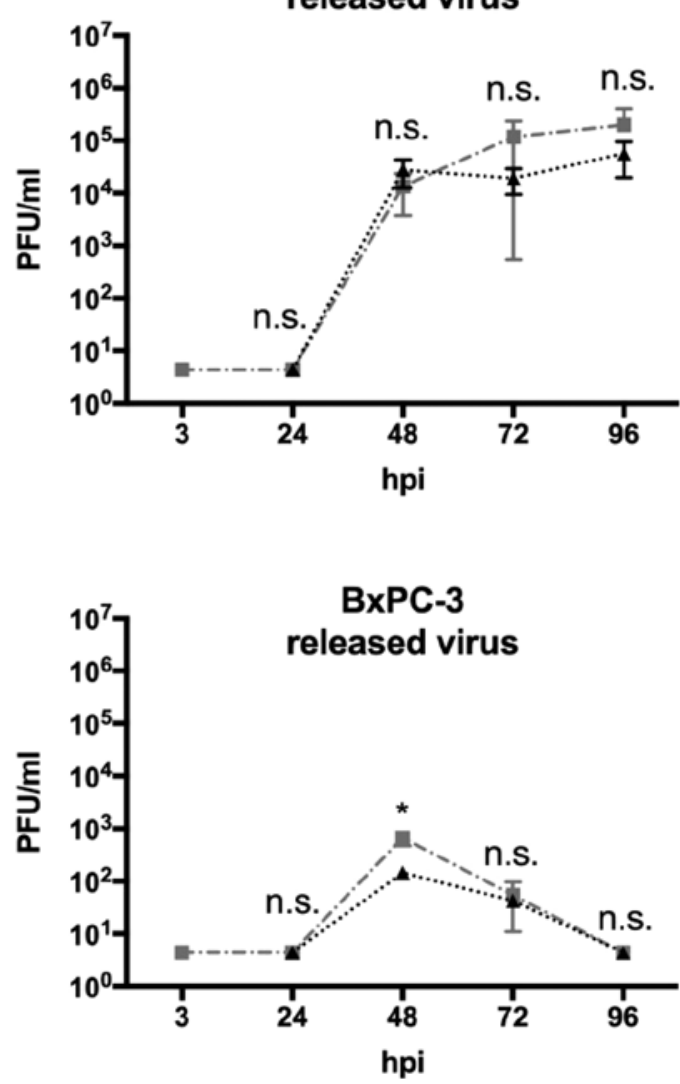

Figure 3. Virus growth curves illustrating the course of viral replication in pancreatic cancer cells infected with oncolytic MeV. Virus growth of MeV-SCD (here denoted as $\mathrm{MeV}$ ) was determined both in cell suspensions (continuous line graphs, cell associated virus) as well as in tumor cell culture supernatants (dotted line graphs, released virus). Viral replication was compared between: i) Single treated, i.e., 'only' infected tumor cells (MeV; mono-virotherapy, grey graphs); and ii) chemovirotherapeutic treated tumor cells, being infected first and then treated additionally with gemcitabine at $3 \mathrm{hpi}(\mathrm{MeV}+\mathrm{Gem}$, black graphs). Notably, tumor-cell specific multiplicities of infection of $\mathrm{MeV}$ and concentrations of Gem were used equal to the concentrations employed before in the viability assays (Sulforhodamin B and MTT). Except for the 48-h-value of released virus in the cell line BXPC-3, there was no statistically significant difference between the values of viral growth with or without Gem. All statistical analyses were conducted with Bonferroni's multiple comparison test. ${ }^{*} \mathrm{P}<0.05$ vs. MeV-Gem. PFU, plaque forming units; n.s., not significant; hpi, hours post infection; GEM, gemcitabine; MeV, measles vaccine virus.

and concentrations of gemcitabine were the same as applied for SRB and MTT assays before.

As a result, viral replication was found to be quite similar for both conditions: Mono-virotherapy or combined chemovirotherapy. For two human pancreatic carcinoma cell lines (MIA PaCa-2 and BxPC-3) viral replication was slightly inhibited in the course of an additional treatment with gemcitabine (Fig. 3): In MIA PaCa-2, cell associated viral titers at $72 \mathrm{~h}$ post infection (hpi) were $8 \times 10^{6}$ plaque forming units (PFU) for $\mathrm{MeV}-\mathrm{SCD}$ alone and $1 \times 10^{6} \mathrm{PFU}$ for the chemovirotherapeutic combination. In BxPC-3, cell associated viral titers at $48 \mathrm{hpi}$ were $1.6 \times 10^{3} \mathrm{PFU}$ for $\mathrm{MeV}-\mathrm{SCD}$ and $0.17 \times 10^{3} \mathrm{PFU}$ for the chemovirotherapeutic combination. It must be pointed out that cell viabilities very likely were reduced due to addition of the chemotherapeutic compound gemcitabine (MOIs of MeV-SCD were the same for both conditions). Furthermore, 

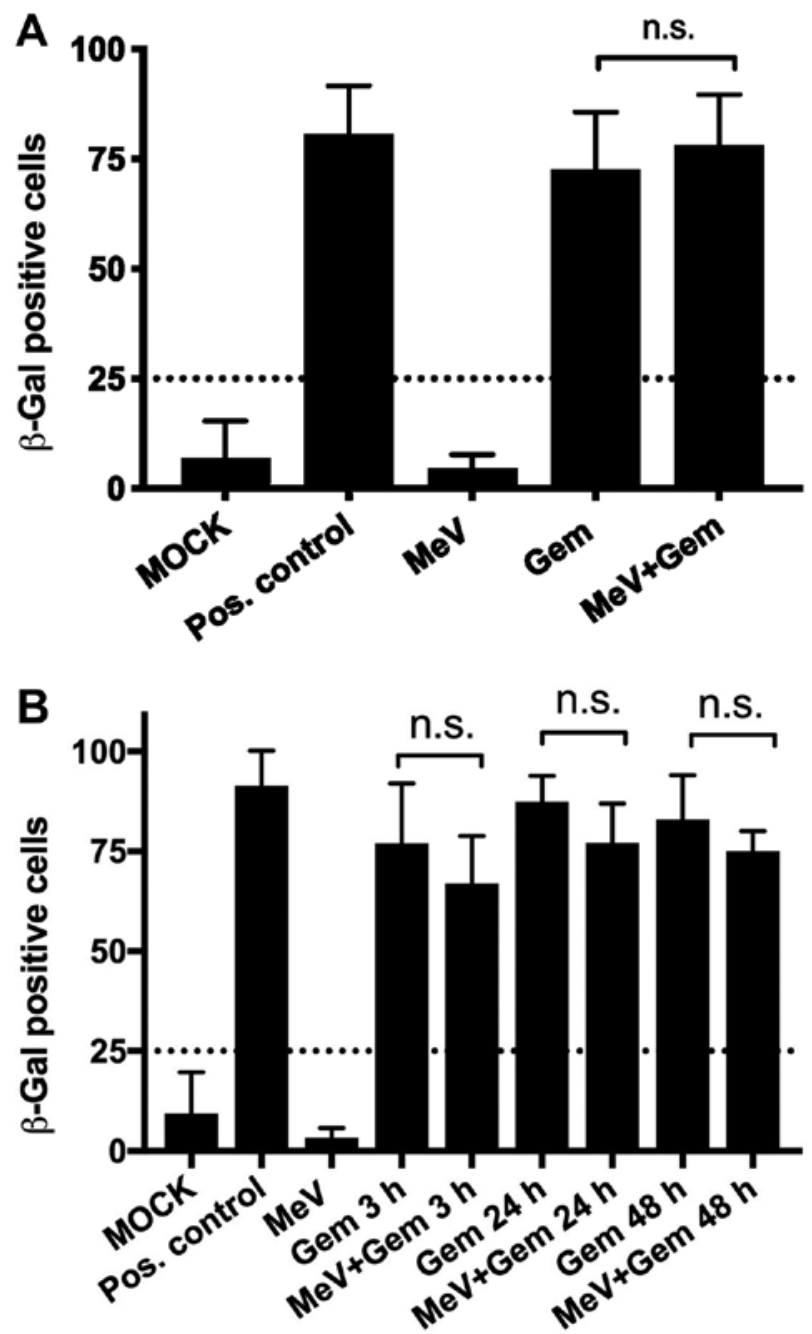

Figure 4. SA- $\beta$-Gal assay illustrating therapy-induced senescence in MIA $\mathrm{PaCa}-2$ cells. (A) Tumor cells were either infected with the oncolytic MeV-SCD (here denoted as $\mathrm{MeV}$ ) or treated with Gem at $3 \mathrm{hpi}$, and underwent combined chemovirotherapeutic treatment $(\mathrm{MeV}+\mathrm{Gem})$ or were left untreated (MOCK); pos. control: Gem $100 \mu \mathrm{M}$; then, expression of SA- $\beta$-gal was determined $72 \mathrm{~h}$ later. Statistical analyses were conducted with an unpaired t-test, confidence interval 95\% and two-tailed). (B) Time dependency of senescence induction. $\operatorname{Gem}(0.03 \mu \mathrm{M})$ was added either at 3,24 or 48 hpi. Expression of SA- $\beta$-gal was determined again at $72 \mathrm{hpi}$. There was no statistically significant impairment of induction of senescence by the presence of virus. Statistical analyses were conducted with Bonferroni's multiple comparison test. hpi, hours post infection; SA- $\beta$-Gal, senescence-associated $\beta$-galactosidase; Gem, gemcitabine; hpi, hours post infection; pos. control, positive control; n.s., not significant; $\mathrm{MeV}$, measles vaccine virus.

viral replication in $\mathrm{BxPC}-3$ was rather low compared to the other two cell lines and did not exceed $10^{4}$ PFU. To further investigate this phenomenon in more detail, cell numbers seeded per well were raised to $2 \times 10^{5}$ cells and $3 \times 10^{5}$ per well, respectively (data not shown). However, the extent of virus replication was not altered significantly by merely elevating the cell numbers.

Therapy-induced senescence (TIS) in MeV-infected tumor cells. To investigate whether the effect of gemcitabine comprises foremost cytotoxicity or additionally senescence in MIA PaCa-2, human pancreatic carcinoma cells infected with MeV-SCD, a senescence-associated $\beta$-galactosidase (SA- $\beta$-Gal) assay was performed (Fig. 4). Interestingly, gemcitabine was found to induce senescence both in uninfected and in infected MIA PaCa- 2 cells with the same potency (Fig. 4A; 70-80\% senescent cells when normalized to the total cell count), when gemcitabine was applied in concentrations being identical to the ones used for the chemovirotherapeutic combination experiments before $(0.03 \mu \mathrm{M})$.

Compared to the positive control (gemcitabine $100 \mu \mathrm{M}$ ), it revealed the same effectiveness in inducing senescence in the remaining cells.

Tumor cell mass was not only significantly reduced by combination of MeV-SCD and gemcitabine as shown in Fig. 2B but also consisted mostly of senescent cells, which are in a permanent cell cycle arrest.

To determine whether the time point of the addition of gemcitabine after the infection with $\mathrm{MeV}-\mathrm{SCD}$ was crucial for the induction of senescence another setting for the SA- $\beta-G a l$ assay was chosen (Fig. 4B). The add-on of gemcitabine was delayed either 24 or $48 \mathrm{~h}$ after infection with MeV-SCD to allow the virus to infect and replicate efficiently before the onset of TIS. As a result, the percentage of senescent cells was found to range between 70 and $90 \%$ independently of the time span between infection and additional treatment with gemcitabine. Accordingly, no significant differences in the time courses of TIS-induction were observed.

Visualization of a contemporaneous presence of senescence and MeV-infection in the same tumor cells. To reassure that senescence and infection with $\mathrm{MeV}$ did not only occur as side-by-side phenomena but simultaneously in the same, identical cells, we microscopically investigated whether $\mathrm{MeV}$-infected MIA-PaCa-2 tumor cells were also positive for SA- $\beta$-Gal staining. For this purpose tumor cells were infected with MeV-GFP (MOI 0.4), a MeV encoding a green fluorescent marker protein (GFP), which helps to detect infected tumor cells easily by fluorescence microscopy, and then again treated with gemcitabine $(0.03 \mu \mathrm{M})$ at $3 \mathrm{hpi}$. As a result, senescence became clearly visualized by the light blue color being detected in the respective SA- $\beta$-Gal assay (Fig. 5A) as well as by an enlarged and flattened cellular phenotype (Fig. 5C), whereas infection with MeV-GFP was visualized by fluorescence microscopy (Fig. 5B). Furthermore, MeV-induced multinucleated syncytia (GFP-positive; Fig. 5E) were also found to undergo senescence (Fig. 5D and E) in the course of addition of gemcitabine at $3 \mathrm{hpi}$.

\section{Discussion}

$\mathrm{MeV}$ constitutes a novel biological compound to overcome therapeutic resistance of pancreatic cancer, despite the fact that anti-viral resistances (primary/secondary) do exist or arise (23). Therefore, novel combination strategies to treat pancreatic cancer have to be developed. As gemcitabine constitutes a first-line therapeutic for the treatment of pancreatic cancer (e.g., in combination therapy with the tyrosine kinase inhibitor EGFR-TKI), we investigated whether the combination of $\mathrm{MeV}$ with that very agent showed superior effects when compared to either agent alone.

Cell viability assays showed a superior cytotoxic effect for the combination of $\mathrm{MeV}$ and gemcitabine when compared to both therapeutics administered as single agents alone. These 
A

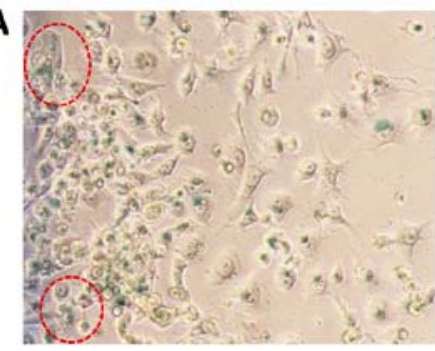

D

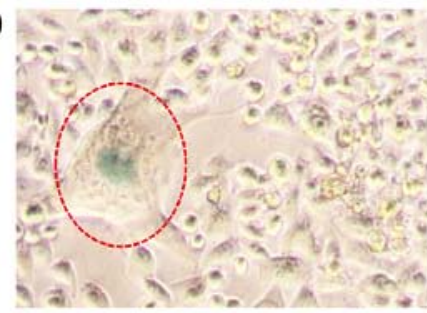

B

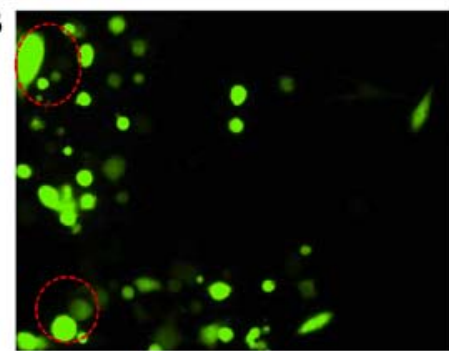

E

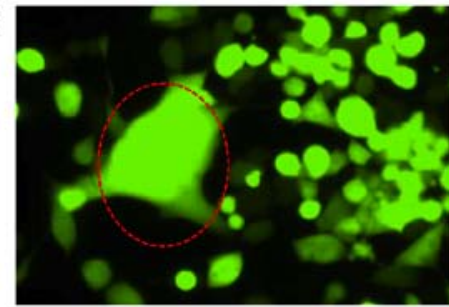

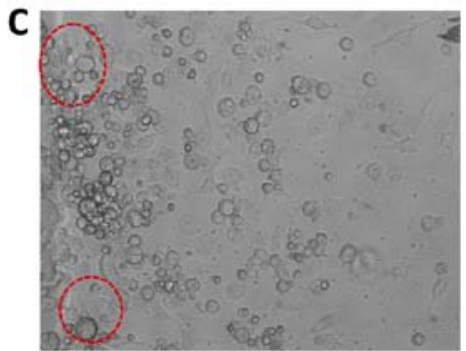

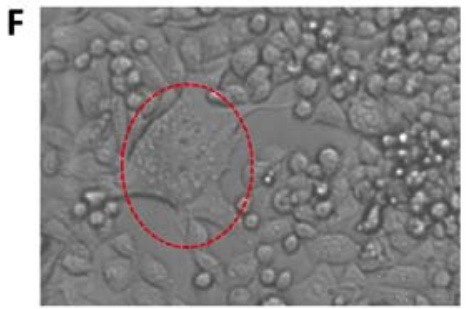

Figure 5. Senescence patterns induced by gemcitabine in pancreatic cancer cells infected with oncolytic MeV. Upper panel (all magnification, $\mathrm{x} 4$ ): (A) MIA $\mathrm{PaCa}-2$ tumor cells infected with the GFP marker gene encoding oncolytic MeV (MeV-GFP) exhibited blue staining of senescence-associated $\beta$-galactosidase, detected by bright field microscopy. (B) Visualization of MeV-GFP infected tumor cells by fluorescence microscopy. (C) Light microscopy of the same sector exhibiting an enlarged and flattened phenotype of tumor cells, being characteristic for the induction of therapy-induced senescence. Red dotted circles indicate examples of MeV-GFP infected senescent tumor cells. Lower panel (all magnification, x10): (D) Higher magnification of a MeV (MeV-GFP) induced syncytium of MIA-PaCa-2 tumor cells exhibiting a blue colored ( $\beta$-galactosidase positive) senescent phenotype. (E) Proving infection with MeV-GFP, this syncytium (encircled in red) exhibited a strong GFP-mediated fluorescence signal. (F) Light microscopy depicted the multinucleated phenotype being typical for MeV-induced syncytia. Tumor cells (MIA PaCa-2) were treated with the respective concentrations used in the combination experiments (MOI of MeV: 0.4; concentration Gem: $0.03 \mu \mathrm{M}$ ), pictures were taken at 72 hpi. Gem, gemcitabine; hpi, hours post infection; MeV, measles vaccine virus; GFP, green fluorescent protein.

results are also interesting for a possible transfer to clinical scenarios considering that lower drug concentrations lead to lower side-effects, as already shown in combination with another oncolytic virus, i.e., Myxoma virus (24). Two different assays were performed, SRB assay measuring cell mass and MTT assay measuring metabolic activity of cells. For the tumor cell lines MIA PaCa-2 and BxPC-3 results of both assays were found to be quite similar. In contrast, for PANC-1 tumor cells, when treated solely with gemcitabine, we observed a significant difference between the results of the SRB and the MTT assay (Fig. 2B; third pair of black and white bars from the left): Cell viability in the MTT assay was about $100 \%$, whereas cell mass in the SRB assay was measured less than $80 \%$ in comparison to the control. A likely explanation for that difference is the senescence-inducing potency of gemcitabine. Cell metabolism in senescent cells remains active, which is measured by the MTT assay. Interestingly, cell viability of the combination of both $\mathrm{MeV}$ and gemcitabine was not found to differ between both assays.

The phenomenon of senescence is of high interest for the field of cancer therapy and is considered as one of the major stress responses to cancer therapies, e.g., to chemotherapeutic compounds $(25,26)$. However, senescent cells can escape the senescence-associated cell cycle arrest or secrete soluble factors that might eventually exert pro-tumorigenic effects on neighboring cells. Accordingly, it is required to develop senolytic regimens which are instrumental in ablating such senescent cells before any of these escape or tumor-inducing events could occur. In this way, OVs should be tested thoroughly in combination with chemotherapeutic compounds for their senolytic potentials, respectively. Several drugs-including gemcitabine-are able to induce senescence in tumor cells resulting in a permanent cell cycle arrest and consequently maintaining cells in a less malignant/less proliferative state (17). In a previous study we could show that $\mathrm{MeV}$ can infect, replicate in and lyse senescent cells including pancreatic cancer cells even more efficiently than non-senescent cells (17). In this context, we investigated whether senescence can be induced in 'already' MeV-infected tumor cells.

For this purpose, we first infected pancreatic cancer cells with $\mathrm{MeV}$ and then added gemcitabine at several different time points up to $48 \mathrm{hpi}$ with $\mathrm{MeV}$. The results indicate senescence-induction is not altered by infection with $\mathrm{MeV}$, independently of the time point of the addition of gemcitabine. In summary these findings suggest that senescence and $\mathrm{MeV}$ infection are not inconsistent cellular mechanisms when cytotoxicity is the target. No matter which mode of application was chosen concerning the sequence of administration, induction of senescence led to an increased oncolytic cell death when compared to $\mathrm{MeV}$ infection alone.

Similar results already have been obtained for the combination of the oncolytic Coxsackievirus A21 in combination with the senescence inducing agent doxorubicine. Simultaneous application of virus and drug as well as infection and addition of the chemotherapeutic compound $24 \mathrm{~h}$ later showed synergistic effects concerning cytotoxicity. No influence of doxorubicine on viral replication was observed (27). Gemcitabine has already been co-administered with different OVs. In one study pancreatic carcinoma cell lines were treated with gemcitabine in combination with myxoma virus (MYXV). The drug was found to inhibit viral gene expression upon simultaneous administration. Sequential treatment, however, resulted in a striking decrease in tumor cell viability when compared to the respective monotherapies. Interestingly, the optimal sequence (drug first or 
virus first) was found to be dependent on the tumor cell line to be addressed (24). Gemcitabine also was shown to increase the oncolytic efficiency of parvovirus H-1PV in pancreatic carcinoma cells when administered $24 \mathrm{~h}$ before the virus (28).

Viral replication is a crucial mechanism for the efficiency of $\mathrm{OV}$ as it ensures a multiplication of the oncolytic potency as well as further spreading of viral particles. Thus, the influence of gemcitabine on viral replication was an important aspect to investigate. In our setting, we observed a slight decrease in viral replication when gemcitabine was administered additionally (Fig. 2). In another study, oncolytic herpes simplex virus type 1 (HSV-1) was shown to replicate better in the presence of gemcitabine (29). However, a different application scheme was used with gemcitabine being added $6 \mathrm{~h}$ prior to infection. The reduced tumor cell number due to the cytotoxic effect of gemcitabine might have caused the reduced viral replication in our work, as the presence of vital cells is crucial for the replication of $\mathrm{MeV}$. Direct alteration of viral replication by gemcitabine is also very likely as gemcitabine was originally developed as anti-viral therapy and shows anti-viral activity against numerous RNA and DNA viruses (30). Of note, viral replication was only decreased but not suppressed and cytotoxicity was significantly higher with $\mathrm{MeV}$ than with gemcitabine alone. Thus, we can deduce that an anti-viral activity of gemcitabine is very likely but the efficiency is not high enough to suppress the cytotoxic mechanism of action of oncolytic MeVs.

Combination of gemcitabine and $\mathrm{MeV}$ constitutes a reasonable new approach to overcome therapeutic resistance of pancreatic cancer cells in vitro. We were able to show that a combination of suboptimal concentrations of both therapeutics led to a significant increase in cytotoxicity in three tumor cell lines when compared to single agent treatment. It remains to be determined, whether there is a distinct molecular pathway that leads to the observed combinatorial effect. However, both therapeutics were shown to work well together and did not alter significantly efficacy of each other. For further research, it is indispensable to transfer our findings to in vivo scenarios in order to learn more on possible obstacles and the most advantageous way to apply these therapeutics. It is very important to optimize delivery of therapeutics especially in pancreatic cancer, as it is hard to reach pancreatic tumor tissues via systemic approaches due to its hypoxia as described earlier. Therefore, alternative modes of application should be tested in vivo, e.g., intratumoral or intraperitoneal (i.p.) routes (31).

Furthermore, new findings concerning tumor biology should be taken into consideration searching for other therapeutic options. In that context, it is very important to analyze different therapeutic regimens (32), and the influence of the immune system on virotherapy-considering both 'negative' aspects as it weakens viral infection and 'positive' aspects as the activation of the immune system leads to anti-tumor immune defense $(7,18)$. In a recently published review the necessity of a very profound investigation and understanding of tumor biology was pointed out as well (33). Moreover, it was accented that identification of synergistically working chemovirotherapy is urgently needed-aiming not only for an exploitation of cytopathic effects but also for an alteration of the immunosuppressive microenvironment of pancreatic cancer $(34,35)$.

\section{Acknowledgements}

The authors would like to thank Mrs. Irina Smirnow [Department of Internal Medicine VIII (Medical Oncology and Pneumology), University Hospital Tuebingen, Tuebingen, Germany], Mrs. Andrea Schenk [Department of Vegetative and Clinical Physiology, University Hospital Tuebingen, Tuebingen, Germany], Mrs. Christine Geisler [Department of Internal Medicine I (Gastroenterology, Gastroenterologic Oncology, Hepatology, Infectiology and Geriatric Medicine), University Hospital Tuebingen, Tuebingen, Germany], Dr Julia Beil [Department of Internal Medicine VIII (Medical Oncology and Pneumology), University Hospital Tuebingen], Dr. Martina Schell (Hain Lifescience GmbH, Nehren, Germany) and Dr Frank Essmann (Interfaculty Institute of Biochemistry, Tuebingen, Germany) for their support of this work.

\section{Funding}

The present study was supported by the intramural IZKF-scholarship of the Faculty of Medicine (University of Tuebingen) awarded to VM, PASCOE pharmazeutische Praeparate $\mathrm{GmbH}$, a grant from the Else-Uebelmesser-Stiftung (grant no. D3021947), the German Research Council (Deutsche Forschungsgemeinschaft) and the Open Access Publishing Fund of University of Tuebingen.

\section{Availability of data and materials}

The datasets used and/or analyzed during the present study are available from the corresponding author on reasonable request.

\section{Authors' contributions}

UML conceived and designed the study, interpreted the data and reviewed the manuscript. VM performed the experiments and was a major contributor in writing the manuscript. SB contributed to the experiments and the interpretation of the data as well as writing the manuscript. AB, SV and CL assisted with the senescence experiments and the interpretation of the SRB viability assay. MB and SV analyzed and interpreted the data. NPM interpreted the data and reviewed the manuscript.

\section{Ethics approval and consent to participate}

Not applicable.

\section{Patient consent for publication}

Not applicable.

\section{Competing interests}

The authors declare that they have no competing interests.

\section{References}

1. Stathis A and Moore MJ: Advanced pancreatic carcinoma: Current treatment and future challenges. Nat Rev Clin Oncol 7: 163-172, 2010. 
2. Huang P, Chubb S, Hertel LW, Grindey GB and Plunkett W: Action of 2',2'-difluorodeoxycytidine on DNA synthesis. Cancer Res 51: 6110-6117, 1991.

3. Gresham GK, Wells GA, Gill S, Cameron C and Jonker DJ: Chemotherapy regimens for advanced pancreatic cancer: A systematic review and network meta-analysis. BMC Cancer 14: 471, 2014

4. Vacchelli E, Eggermont A, Sautès-Fridman C, Galon J, Zitvogel L, Kroemer G and Galluzzi L: Trial watch: Oncolytic viruses for cancer therapy. Oncoimmunology 2: e24612, 2013.

5. Sze DY, Reid TR and Rose SC: Oncolytic virotherapy. J Vasc Interv Radiol 24: 1115-1122, 2013.

6. Forbes NE, Krishnan R and Diallo JS: Pharmacological modulation of anti-tumor immunity induced by oncolytic viruses. Front Oncol 4: 191, 2014.

7. Chiocca EA and Rabkin SD: Oncolytic viruses and their application to cancer immunotherapy. Cancer Immunol Res 2: 295-300, 2014

8. Nakao A, Kasuya H, Sahin TT, Nomura N, Kanzaki A, Misawa M, Shirota T, Yamada S, Fujii T, Sugimoto H, et al: A phase I dose-escalation clinical trial of intraoperative direct intratumoral injection of HF10 oncolytic virus in non-resectable patients with advanced pancreatic cancer. Cancer Gene Ther 18: 167-175, 2011.

9. Hecht JR, Bedford R, Abbruzzese JL, Lahoti S, Reid TR, Soetikno RM, Kirn DH and Freeman SM: A phase I/II trial of intratumoral endoscopic ultrasound injection of ONYX-015 with intravenous gemcitabine in unresectable pancreatic carcinoma. Clin Cancer Res 9: 555-561, 2003.

10. Mulvihill S, Warren R, Venook A, Adler A, Randlev B, Heise $C$ and Kirn D: Safety and feasibility of injection with an E1B-55 kDa gene-deleted, replication-selective adenovirus (ONYX-015) into primary carcinomas of the pancreas: A phase I trial. Gene Ther 8: 308-315, 2001.

11. Wennier S, Li S and McFadden G: Oncolytic virotherapy for pancreatic cancer. Expert Rev Mol Med 13: e18, 2011.

12. Russell SJ, Federspiel MJ, Peng KW, Tong C, Dingli D, Morice WG, Lowe V, O'Connor MK, Kyle RA, Leung N, et al Remission of disseminated cancer after systemic oncolytic virotherapy. Mayo Clin Proc 89: 926-933, 2014.

13. Carlson SK, Classic KL, Hadac EM, Dingli D, Bender CE Kemp BJ and Russell SJ: Quantitative molecular imaging of viral therapy for pancreatic cancer using an engineered measles virus expressing the sodium-iodide symporter reporter gene. AJR Am J Roentgenol 192: 279-287, 2009.

14. Bossow S, Grossardt C, Temme A, Leber MF, Sawall S, Rieber EP, Cattaneo R, von Kalle C and Ungerechts G: Armed and targeted measles virus for chemovirotherapy of pancreatic cancer. Cancer Gene Ther 18: 598-608, 2011.

15. Ellerhoff TP, Berchtold S, Venturelli S, Burkard M, Smirnow I, Wulff T and Lauer UM: Novel epi-virotherapeutic treatment of pancreatic cancer combining the oral histone deacetylase inhibitor resminostat with oncolytic measles vaccine virus. Int J Oncol 49: 1931-1944, 2016.

16. Awano M, Fujiyuki T, Shoji K, Amagai Y, Murakami Y, Furukawa Y, Sato $H$, Yoneda $M$ and Kai C: Measles virus selectively blind to signaling lymphocyte activity molecule has oncolytic efficacy against nectin-4-expressing pancreatic cancer cells. Cancer Sci 107: 1647-1652, 2016.

17. Weiland T, Lampe J, Essmann F, Venturelli S, Berger A, Bossow S, Berchtold S, Schulze-Osthoff K, Lauer UM and Bitzer M: Enhanced killing of therapy-induced senescent tumor cells by oncolytic measles vaccine viruses. Int J Cancer 134: 235-243, 2014

18. Berchtold S, Lampe J, Weiland T, Smirnow I, Schleicher S, Handgretinger R, Kopp HG, Reiser J, Stubenrauch F, Mayer N, et al: Innate immune defense defines susceptibility of sarcoma cells to measles vaccine virus-based oncolysis. J Virol 87: 3484-3501, 2013.
19. Skehan P, Storeng R, Scudiero D, Monks A, McMahon J, Vistica D, Warren JT, Bokesch H, Kenney S and Boyd MR: New colorimetric cytotoxicity assay for anticancer-drug screening. J Natl Cancer Inst 82: 1107-1112, 1990.

20. Mosmann T: Rapid colorimetric assay for cellular growth and survival: Application to proliferation and cytotoxicity assays. J Immunol Methods 65: 55-63, 1983.

21. Kärber G: Beitrag zur kollektiven Behandlung pharmakologischer Reihenversuche. Archiv für Experiment Pathol und Pharmakol 162:480-483, 1931.

22. Spearman C: The method of 'Right and Wrong Cases' (Constant Stimuli) without Gauss's formula. Br J Psychol 2: 227-242, 1908.

23. Noll M, Berchtold S, Lampe J, Malek NP, Bitzer M and Lauer UM: Primary resistance phenomena to oncolytic measles vaccine viruses. Int J Oncol 43: 103-112, 2013.

24. Wennier ST, Liu J, Li S, Rahman MM, Mona M and McFadden G: Myxoma virus sensitizes cancer cells to gemcitabine and is an effective oncolytic virotherapeutic in models of disseminated pancreatic cancer. Mol Ther 20: 759-768, 2012.

25. Krizhanovsky V, Xue W, Zender L, Yon M, Hernando E and Lowe SW: Implications of cellular senescence in tissue damage response, tumor suppression, and stem cell biology. Cold Spring Harb Symp Quant Biol 73: 513-522, 2008

26. Rodier F and Campisi J: Four faces of cellular senescence. J Cell Biol 192: 547-556, 2011.

27. Skelding KA, Barry RD and Shafren DR: Enhanced oncolysis mediated by Coxsackievirus A21 in combination with doxorubicin hydrochloride. Invest New Drugs 30: 568-581, 2012.

28. Angelova AL, Aprahamian M, Grekova SP, Hajri A, Leuchs B, Giese NA, Dinsart C, Herrmann A, Balboni G, Rommelaere J and Raykov Z: Improvement of gemcitabine-based therapy of pancreatic carcinoma by means of oncolytic parvovirus H-1PV. Clin Cancer Res 15: 511-519, 2009.

29. Eisenberg DP, Adusumilli PS, Hendershott KJ, Yu Z, Mullerad M, Chan MK, Chou TC and Fong Y: 5-fluorouracil and gemcitabine potentiate the efficacy of oncolytic herpes viral gene therapy in the treatment of pancreatic cancer. J Gastrointest Surg 9: 1068-1079, 2005.

30. Hertel LW, Boder GB, Kroin JS, Rinzel SM, Poore GA, Todd GC and Grindey GB: Evaluation of the antitumor activity of gemcitabine (2',2'-difluoro-2'-deoxycytidine). Cancer Res 50: 4417-4422, 1990.

31. Wang H, Chen NG, Minev BR, Zimmermann M, Aguilar RJ, Zhang Q, Sturm JB, Fend F, Yu YA, Cappello J, et al: Optical detection and virotherapy of live metastatic tumor cells in body fluids with vaccinia strains. PLoS One 8: e71105, 2013.

32. Yurttas C, Berchtold S, Malek NP, Bitzer M and Lauer UM: Pulsed versus continuous application of the prodrug 5-fluorocytosine to enhance the oncolytic effectiveness of a measles vaccine virus armed with a suicide gene. Hum Gene Ther Clin Dev 25: 85-96, 2014.

33. Singh HM, Ungerechts G and Tsimberidou AM: Gene and cell therapy for pancreatic cancer. Expert Opin Biol Ther 15: 505-516, 2015.

34. Zamarin D, Holmgaard RB, Subudhi SK, Park JS, Mansour M, Palese P, Merghoub T, Wolchok JD and Allison JP: Localized oncolytic virotherapy overcomes systemic tumor resistance to immune checkpoint blockade immunotherapy. Sci Transl Med 6: 226ra32, 2014.

35. Engeland CE, Grossardt C, Veinalde R, Bossow S, Lutz D, Kaufmann JK, Shevchenko I, Umansky V, Nettelbeck DM, Weichert W, et al: CTLA-4 and PD-L1 checkpoint blockade enhances oncolytic measles virus therapy. Mol Ther 22: 1949-1959, 2014 